ihre Eigenständigkeit verzichten. Die Vorteile seien aber nahe liegend: „Die Ärzte haben eine gemeinsame Patientenakte“, erklärt Leutloff. So kann jeder Behandler direkt auf die Patientendaten zugreifen. „Doppeluntersuchungen lassen sich besser vermeiden, weil man etwa sieht, der Kollege hat schon ein Röntgenbild anfertigen lassen. " Der Mitbehandler sehe zudem, welche Medikamente verordnet wurden. „So kann unerwünschten Wechselwirkungen vorgebeugt werden." Und der Patient hat feste Ansprechpartner - auch wenn sein Hausarzt einmal im Urlaub ist. Leutloff: "Gerade wenn ich ein multimorbider $\mathrm{Pa}$ tient bin und regelmäßig mehrere Ärzte aufsuchen muss, hat es Vorteile, wenn ich in eine BAG oder ein MVZ gehe, wo die Versorgung eng abgestimmt ist.“ Praxen sparen hier eindeutig Kosten und der einzelne Arzt hat dadurch, dass
Verwaltungsaufgaben zusammengelegt werden, mehr Zeit für die Patienten.

\section{Der Patient in ganzheitlicher Betrachtung}

Die Patienten profitieren auch deshalb von der Versorgung aus einer Hand, weil sie in dieser Versorgungsform ganzheitlich gesehen werden. „Wenn ich zu einem Facharzt gehe, konzentriert er sich oftmals auf einen bestimmten Aspekt der Erkrankung“, so Leutloff. In kooperativen Versorgungsformen betrachten aber verschiedene Ärzte mit unterschiedlichem Blickwinkel den Patienten, sodass eher ein ganzheitliches Bild entstehe, bzw. auch der Patient und sein Fall mit all seinen Facetten betrachtet werde.

Auch über Ärztenetze lässt sich die „Versorgung aus einer Hand“ gut gestalten. Hier bleiben die Praxen in jedem Fall selbstständig - trotzdem gibt es lo-

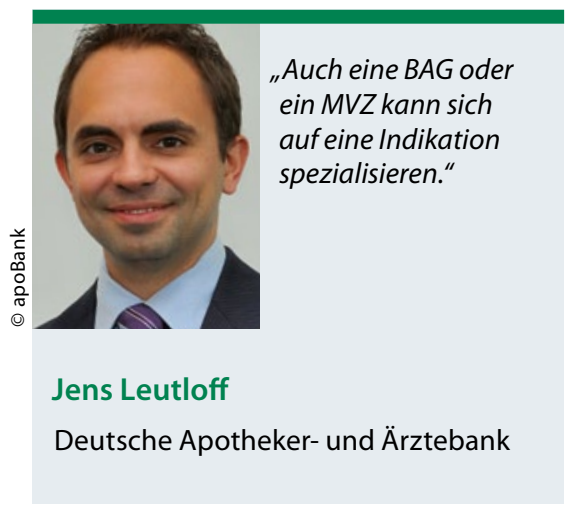

sere und engere Verbünde. Wer enger zusammenrückt, kann etwa die gemeinsame elektronische Patientenakte nutzen. Der Vorteil von Netzen: Als größerer Verbund können sie leichter Einzelversorgungsverträge mit den Kassen aushandeln.

Rebekka Höhl

\title{
Ambulant und stationär - Kooperation an der Schnittstelle
}

\author{
Die Ausgestaltung der ambulanten spezialfachärztlichen Versorgung steht noch immer in den Sternen. \\ Was tut sich nun an der Schnittstelle ambulant/stationär?
}

$\mathrm{H}$ offnungen, die medizinische Versorgung sektorenübergreifend zu verbessern, ruhten lange Zeit vor allem auf Integrationsverträgen (IV). Doch seit dem Auslaufen der Anschubfinanzierung Ende 2008 ist es um neue IV-Projekte still geworden. „Viele Ärztenetze haben überzeugende Projekte in der Schublade“, weiß Jens Leutloff von der Deutschen Apotheker- und Ärztebank (apoBank), „doch sie finden keine Vertragspartner, weil die Kassen das Risiko scheuen“. Effekte auf die Ausgaben etwa durch Vermeidung von Krankenhauseinweisung - ließen sich oft erst mittel- bis langfristig nachweisen. Höhere Kosten beim Start von IV-Projekten erhöhten dagegen die Gefahr, einen $\mathrm{Zu}$ satzbeitrag fordern zu müssen.

„Aus finanziellen Gründen haben es neue IV-Projekte momentan schwer. Aber das muss nicht so bleiben." Es werde inzwischen auch wieder über einen Innovationsfonds diskutiert, so Leutloff. Daher räumt er der IV durchaus auch in Zukunft Chancen ein. Aktuell aber ste- he die ambulante spezialfachärztliche Versorgung (ASV) im Mittelpunkt des Interesses, wenn von sektorenübergreifender Zusammenarbeit oder Versorgung aus einer Hand die Rede ist. Zwar liegt hierzu noch vieles im Unklaren. Eigentlich sollte diese neue Versorgungsebene, bei der niedergelassene Fachärzte und Kliniken möglichst kooperieren sollen, Anfang 2013 Wirklichkeit werden. Doch derzeit ist völlig offen, wann der G-BA die geforderten Richtlinien zur Konkretisierung der ASV fertig stellen kann. Der G-BA, so Leutloff, soll bestimmen, welche Erkrankungen mit besonderen Krankheitsverläufen und welche Art schwerer Verlaufsformen für die ASV in Frage kommen. Darüber hinaus soll er seltene Erkrankungen sowie hochspezialisierte Leistungen definieren. Außerdem kann er Regelungen für die Vertragsbeziehungen zwischen niedergelassenen Fachärzten und ortsansässigen Kliniken treffen.

Für die Versorgung von Krebspatienten sind solche Vereinbarungen im Ver- sorgungsstrukturgesetz verbindlich vorgeschrieben. Leutloff erwartet deshalb auch, dass einer der Schwerpunkte der ambulanten spezialfachärztlichen Versorgung in der Onkologie liegen wird. Weitere Aufgaben des G-BA im Kontext der ASV sind Vorschriften zur Qualitätssicherung und zu den personellen wie materiellen Voraussetzungen.

\section{Gute Prognose für ASV}

Obwohl es auf all diese Fragen noch keine Antwort gibt, ist Leutloff überzeugt, dass die ASV für niedergelassene Fachärzte erfolgversprechend ist. „Schon wegen der extrabudgetären Vergütung macht die Teilnahme Sinn. Aber auch den Patienten, die keinen Arztwechsel mehr zwischen ambulanter und stationärer Ebene verkraften müssen, verspricht die ASV einige Verbesserungen." Von daher werde das Interesse daran „sicher groß sein“. Zumal die Teilnahme einfacher ist, als bei der integrierten Versorgung, die ohne einen Vertragspartner aus dem Kassen-lager nicht geht. Christoph Winnat 